\title{
EVALUATION OF ORAL HEALTH STATUS OF CHILDREN IN PRETREATMENT AND AFTER TREATMENT FOR 18 MONTH
}

\author{
K. Görkem Ulu Güzel', Melis Akyıldız', Gülçin Doğusal', Sultan Keleş ${ }^{1}$, Işı Sönmez ${ }^{1}$ \\ 'Department of Paediatric Dentistry, Faculty of Dentistry, Adnan Menderes University, Aydin, Turkey \\ ${ }^{2}$ Department of Paediatric Dentistry, Faculty of Dentistry, Bezmialem Foundation University, İstanbul, Turkey
}

\section{SUMMARY}

Objective: Dental caries is an important public health issue worldwide. In developing countries preventive dentistry is not common; hence, oral and dental health problems continue to generate serious economic and social issues. The aim of this study was to assess oral health and the incidence of dental caries in systemically healthy children aged 3-14 years, provide education on oral hygiene motivation, conduct the necessary preventive and restorative procedures, and reassess caries development at the 6-, 12-, and 18-month post-treatment follow-up.

Methods: Systemically healthy children aged 3-14 years who applied to the Paediatric Dentistry Clinic, Faculty of Dentistry, Adnan Menderes University (ADU) were included in the study. The subjects were evaluated for oral health status and incidence of dental caries based on surveys and clinical examination, motivation for oral hygiene with necessary training, completion of preventive and restorative treatments, and development of dental caries at the 6-, 12-, and 18-month post-treatment follow-up.

Results: The study included 320 patients, aged $3-14$ years (mean age $8.08 \pm 3.45$ years). In children included in the study, the average decayed (d), missing ( $\mathrm{m}$ ) and filled (f) primary teeth (dmft) value was 3.81 (min-max: 0-16), decayed (D), missing (M) and filled (F) permanent teeth (DMFT) value, 1.49 (min-max: 0-6), decayed (d), missing(m) and filled(f) primary teeth surface (dmfs) value, 10.11 (min-max: 0-40), and decayed (D), missing (M) and filled (F) permanent teeth surface (DMFS) value, 2.09 (min-max: 0-12).

Conclusions: For the protection and maintenance of oral and dental health in dentistry, preventive approaches should be the first concern and conservative therapeutic methods should be given priority after occurrence of any dental pathology. In addition, one-time examinations are not sufficient, and children should be examined at least twice a year. This study showed that the incidence of dental caries declined in children who underwent periodical examination and preventive dental treatment.

Key words: dental caries, preventive dental treatment, oral hygiene, socioeconomic level

Address for correspondence: K. Görkem Ulu Güzel, Adnan Menderes University, Faculty of Dentistry, Department of Paediatric Dentistry, Kepez Mevkii Efeler, 09100 Aydin, Turkey. E-mail: gorkemulu@yahoo.com

https://doi.org/10.21101/cejph.a5079

\section{INTRODUCTION}

Dental caries is one of the most common diseases in humans. In order to control this prevalent health concern, it is first necessary to spread awareness of prevention and accordingly support with the optimum treatment. Several studies to measure the prevalence, severity of dental caries, and treatment needs have been conducted globally since the mid-21st century (1-4). Acquiring nation-wide data on dental caries and treatment requirement will ensure that the goals are met by setting the grounds for determining policies to improve the health status and deliver preventive and restorative treatments. However, in developing countries that lack preventive dentistry practices, such as Turkey, oral and dental health problems constitute serious economic and social problems $(5,6)$.

Preventive dentistry in children is the practice of caring for existing teeth before they develop caries. Thus, it ensures that the child is nourished better, able to speak clearly and understandably, and finally, has a positive impact on the child's overall health. Parents may provide their children with lifetime oral-dental health if they can promote brushing teeth and using dental floss, regulate their dietary habits, fluoride applications, and regular visits to the dentist.

The purpose of our study was to assess oral health and the incidence of dental caries in systemically healthy children aged 3-14 years, motivate oral hygiene practice with trainings, meet the requirements of preventive and restorative treatments, and analyze caries development up to the 18-month follow-up.

\section{MATERIALS AND METHODS}

This study included 320 patients in total, who applied to the Adnan Menderes University (ADU) Paediatric Dentistry Clinic between March 2014 and March 2015 before their treatments were initiated. The research was approved by the ADU Clinical Researches Ethical Committee (Decision No. 2014/33) and only children whose parents provided written informed consent were included in the study. Four examiners were trained in order to 
limit the intra-examiner variability. The agreement for all the assessments was in the range of 85 to $95 \%$.

The examiners carried out the examinations in a fully equipped dental clinic using a mirror and probe after the teeth had been dried.

Individualized caries preventive information and oral hygiene instruction were provided for all children and parents as part of the examination, including instructions on reducing high-frequency exposures to obvious and hidden sugars, limiting cariogenic foods to mealtimes, rapidly clearing cariogenic foods from the child's oral cavity, either by tooth brushing or by consumption of protective foods, and restricting sugar-containing snacks, and use of fluoridated toothpaste. Oral hygiene techniques were demonstrated to the preschool and school children and their parents with use of the Hygiene Demonstration Model.

For the assessment of developing tooth caries, information on nutrition and oral hygiene habits as well as socioeconomic status of the family was obtained from the survey forms filled by the parents who had consented to participate in the study. The $\mathrm{dmft}$ and the DMFT scores were recorded before dental treatment. Following the assessment of oral health, routine essential preventive treatments (topical sodium fluor varnish (Duraphat, Colgate-Palmolive, NSW, Australia), fissure sealant treatments (Fissürit F, Voco, Germany)) and restorative treatments (Equia Forte, GC, Tokyo, Japan; Dyract, Dentsply, Konstanz, Germany; Clearfill Majesty, Kuraray Dental Inc, Tokyo Japan) were initiated, and dental caries development was reassessed 6, 12, and 18 months later.

Table 1. Average dmft and DMFT values in children included in the study

\begin{tabular}{|l|c|c|c|}
\hline & Mean & Median & Min-max \\
\hline dmft & 3.81 & 3.00 & $0-16$ \\
\hline DMFT & 1.49 & 1.00 & $0-6$ \\
\hline dmfs & 10.11 & 8.00 & $0-40$ \\
\hline DMFS & 2.09 & 1.00 & $0-12$ \\
\hline
\end{tabular}

Survey forms were evaluated statistically following data entry of the oral assessment forms.

Statistical analyses were performed using the software version 18 (SPSS, Chicago, IL, USA). The variables were investigated using visual and analytical methods to determine whether or not data are normally distributed. Descriptive analyses were presented for non-normally distributed and ordinal variables. The chi-square test was carried out for analysis of the data and the differences between means were tested using the Mann-Whitney $\mathrm{U}$ and Wilcoxon tests. Statistically significant levels of $\mathrm{p}<0.001$ and $\mathrm{p}<0.05$ were adopted.

\section{RESULTS}

The study consisted of 320 patients, aged 3-14 years (mean age, $8.08 \pm 3.45)$, of which, $160(50 \%)$ were girls and $160(50 \%)$ were boys. The initial $\mathrm{dmft} / \mathrm{s}$ and DMFT/S values of the children included in the study according to the dentition stages are presented in Tables 1 and 2. The baseline $\mathrm{dmft} / \mathrm{s}$ and DMFT/S values of the girls and boys are presented in Table 3 .

Based on the assessment of socioeconomic status, timing and frequency of tooth brushing, usage of breast milk and baby formula, period of breastfeeding, duration of using a feeding bottle, type of food consumed with a feeding bottle, number of meals per day, and history of dental treatment, no statistically significant differences were observed among the patients of all socioeconomic groups. The educational level of parents and the results of earlier dental examinations showed significant differences among groups $(\mathrm{p}<0.001)$ (Table 4$)$.

Based on the socioeconomic status, group wise differences were observed in terms of the $\mathrm{dmft} / \mathrm{dmfs}$ parameters. The $\mathrm{dmft} /$ $\mathrm{dmfs}$ values of patients in the low and medium group were not statistically significant; however, a statistical difference was found between the values of medium and high, and low and high groups $(\mathrm{p}<0.001)$. Accordingly, lower initial $\mathrm{dmft} / \mathrm{dmfs}$ values were observed in the group with high socioeconomic status. Similarly, while the initial DMFT/DMFS values were lower in the group

Table 2. Initial values of $\mathrm{dmft} / \mathrm{dmfs}$, DMFT/DMFS according to dentition stage $(N=320)$

\begin{tabular}{|l|c|c|c|c|c|c|c|c|c|}
\hline \multirow{2}{*}{} & \multicolumn{3}{|c|}{ Primary dentition $(\mathrm{n}=96)$} & \multicolumn{3}{c|}{ Mixed dentition $(\mathrm{n}=128)$} & \multicolumn{3}{c|}{ Permanent dentition $(\mathrm{n}=96)$} \\
\cline { 2 - 11 } & Mean & Median & Min-max & Mean & Median & Min-max & Mean & Median & Min-max \\
\hline dmft & 2.88 & 2.50 & $0-10$ & 5.17 & 4.00 & $0-16$ & - & - & - \\
\hline DMFT & - & - & - & 0.35 & 0.00 & $0-4$ & 2.80 & 3.00 & $0-6$ \\
\hline dmfs & 9.06 & 8.00 & $0-32$ & 12.87 & 10.00 & $0-40$ & - & - & - \\
\hline DMFS & - & - & - & 0.58 & 0.00 & $0-12$ & 3.81 & 4.00 & $0-10$ \\
\hline
\end{tabular}

*Due to the lack of permanent teeth in the primary dentition and lack of primary teeth in permanent dentition DMFT/DMFS- dmft/dmfs fields are empty.

Table 3. Initial values of dmft/dmfs, DMFT/DMFS according to gender $(N=320)$

\begin{tabular}{|c|c|c|c|c|c|c|}
\hline & \multicolumn{3}{|c|}{$\operatorname{Girl}(n=160)$} & \multicolumn{3}{|c|}{ Boy $(n=160)$} \\
\hline & Mean & Median & Min-max & Mean & Median & $\operatorname{Min}-\max$ \\
\hline $\mathrm{dmft}$ & 3.05 & 3.00 & $0-12$ & 4.64 & 4.00 & $0-16$ \\
\hline DMFT & 1.44 & 1.00 & $0-6$ & 1.56 & 1.00 & $0-6$ \\
\hline $\mathrm{dmfs}$ & 8.23 & 6.00 & $0-33$ & 12.11 & 10.00 & $0-40$ \\
\hline DMFS & 1.89 & 1.00 & $0-10$ & 2.29 & 1.00 & $0-12$ \\
\hline
\end{tabular}


Table 4. Comparison of differences between socioeconomic status and other parameters by the chi-square test $(N=320)$

\begin{tabular}{|c|c|c|c|c|c|c|c|}
\hline & & \multicolumn{6}{|c|}{ Socioeconomic status } \\
\hline & & Low & Medium & High & Total & $x^{2}$ & $\mathrm{p}$-value \\
\hline \multirow{6}{*}{ Frequency of tooth brushing } & Anytime & 0 & 8 & 10 & 18 & \multirow{6}{*}{4.500} & \multirow{6}{*}{0.809} \\
\hline & One time a day & 13 & 83 & 84 & 180 & & \\
\hline & Two times a day & 6 & 33 & 23 & 62 & & \\
\hline & Three times a day & 0 & 1 & 2 & 3 & & \\
\hline & Sometimes & 4 & 26 & 27 & 57 & & \\
\hline & Total & 23 & 151 & 146 & 320 & & \\
\hline \multirow{3}{*}{$\begin{array}{l}\text { Usage of breast milk and } \\
\text { baby formula }\end{array}$} & Breast milk & 21 & 123 & 122 & 266 & \multirow{3}{*}{2.605} & \multirow{3}{*}{0.626} \\
\hline & Baby formula & 0 & 11 & 7 & 18 & & \\
\hline & Breast milk and baby formula & 2 & 17 & 17 & 36 & & \\
\hline \multirow{6}{*}{ Period of breastfeeding } & Anytime & 0 & 11 & 7 & 18 & \multirow{6}{*}{10.809} & \multirow{6}{*}{0.373} \\
\hline & 3 months & 2 & 4 & 6 & 12 & & \\
\hline & 6 months & 2 & 37 & 24 & 63 & & \\
\hline & 12 months & 12 & 54 & 65 & 131 & & \\
\hline & 18 months & 4 & 27 & 29 & 60 & & \\
\hline & $>18$ months & 3 & 18 & 15 & 36 & & \\
\hline \multirow{6}{*}{$\begin{array}{l}\text { Duration of using a feeding } \\
\text { bottle }\end{array}$} & Any & 2 & 7 & 16 & 25 & \multirow{6}{*}{14.191} & \multirow{6}{*}{0.164} \\
\hline & 3 months & 1 & 11 & 15 & 27 & & \\
\hline & 6 months & 5 & 18 & 20 & 43 & & \\
\hline & 12 months & 11 & 69 & 48 & 128 & & \\
\hline & 18 months & 4 & 36 & 31 & 71 & & \\
\hline & $>18$ months & 0 & 10 & 16 & 26 & & \\
\hline \multirow{2}{*}{$\begin{array}{l}\text { Type of food consumed with } \\
\text { a feeding bottle }\end{array}$} & Water & 4 & 33 & 21 & 58 & \multirow{2}{*}{2.801} & \multirow{2}{*}{0.247} \\
\hline & Whole milk & 19 & 118 & 125 & 262 & & \\
\hline \multirow{4}{*}{ Number of daily meals } & 2 & 0 & 1 & 1 & & \multirow{4}{*}{1.358} & \multirow{4}{*}{0.968} \\
\hline & 3 & 13 & 79 & 85 & & & \\
\hline & 4 & 8 & 53 & 45 & & & \\
\hline & 5 & 2 & 18 & 15 & & & \\
\hline \multirow{5}{*}{$\begin{array}{l}\text { Educational level } \\
\text { of the mother }\end{array}$} & Primary school & 19 & 30 & 1 & 50 & \multirow{5}{*}{284.905} & \multirow{5}{*}{$<0.001$} \\
\hline & Secondary school & 4 & 65 & 6 & 75 & & \\
\hline & High school & 0 & 56 & 28 & 84 & & \\
\hline & University & 0 & 0 & 107 & 107 & & \\
\hline & Postgraduate & 0 & 0 & 4 & 4 & & \\
\hline \multirow{5}{*}{ Educational level of the father } & Primary school & 14 & 4 & 1 & 19 & \multirow{5}{*}{369.725} & \\
\hline & Secondary school & 6 & 65 & 0 & 71 & & \\
\hline & High school & 3 & 80 & 20 & 103 & & $<0.001$ \\
\hline & University & 0 & 2 & 118 & 120 & & \\
\hline & Postgraduate & 0 & 0 & 7 & 7 & & \\
\hline Forlior dental pyaminations & Yes & 7 & 63 & 96 & 166 & 21735 & $<\cap 0 \cap 1$ \\
\hline 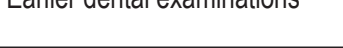 & No & 16 & 88 & 50 & 154 & 21.100 & -0.001 \\
\hline Hictory of dental treatment & Yes & 7 & 46 & 68 & 121 & 8768 & 0012 \\
\hline 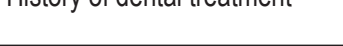 & No & 16 & 105 & 78 & 199 & 0.100 & 0.012 \\
\hline
\end{tabular}

with high socioeconomic status than in the other groups; they were higher in the other groups, with significant difference $(\mathrm{p}<0.001)$.

A reduction in primary teeth caries development of all groups was observed at the 6-month examination, and the lowest value was observed in the group with high socioeconomic status; however, this difference was not statistically significant in patients belonging to the low-middle and middle-high income groups, whereas the difference between the low income and high income 
groups was significant. At the 12-month examination, the $\mathrm{dt} / \mathrm{ds}$ values in patients belonging to the low socioeconomic group were higher, and this difference was statistically significant as compared to the other two groups $(\mathrm{p}<0.001)$. At the 18 -month examination, the $\mathrm{dt} / \mathrm{ds}$ values in the primary teeth were zero.

During the examination of patients at the 6-, 12-, and 18-month follow-up, permanent teeth caries development was not noted in any of the groups.

The pre-school children and school children with regular teeth brushing were $45.8 \%(n=96)$ and $89.7 \%(n=224)$, respectively.

Of children with pre-dental examination, $13.9 \%$ were preschool and $86.1 \%$ were school children. The need for performing dental treatment was identified in $10.4 \%$ of preschool children and $49.6 \%$ of school children.

\section{DISCUSSION}

Dental caries is one of the most common diseases in humans, and the treatment of caries is associated with over cost in developing as well as developed countries. In order to control this prevalent health concern, it is first necessary to spread awareness of prevention measures and accordingly support with the optimum treatment. Informing individuals on oral and dental health, highlighting the importance of preventive programmes, and considering the execution of these programmes as a governmental policy may prevent oral-dental health problems.

Saydam et al. conducted the first research study to determine the level of oral and dental health nation-wide in Turkey in 1988 (7). The study, which has been since adopted as the first representative of the country, led to the report Oral and Dental Health Profiles in Turkey by Güçiz Doğan and Gökalp in 2004 (6). In this study, tooth brushing habits; periodic examinations; and the prevalence of caries (crown-root), filled teeth, missing teeth; mean sum of decayed, missing, filled teeth and DMFT/dmft values were evaluated based on the gender and the residential area. According to the findings of the first study, it was concluded that the WHO goal of the global average for dental caries in 2000 of $\leq 3$ DMFT at 12 years of age was met. The results of the nation-wide study in Turkey showed that the WHO/Europe goal of 2 DMFT at 12 years of age in 2010 was achieved $(\mathrm{DMFT}=1.9)$. In our study, the DMFT $(\mathrm{DMFT}=2.95)$ value for 12-year-olds matches the global goals for 2000 .

The dmft determined for this age group is very high when compared to the values in developed countries. In our study, the mean value of dmft scores for all age groups was 3.81. The highest $\mathrm{dmft}$ score $(\mathrm{dmft}=5.17)$ was observed in mixed dentition.

According to the results of previous studies $(6,7)$ on oral-dental health status in Turkey, although the DMFT score increases from the ages of 12 to 15 years, the DMFT score for age 15 declines from 4.14 in 1988 to 2.3 in 2004; however, the study conducted in 2004 (6) indicates that one of the goals in 2000 regarding "keeping all teeth in $85 \%$ of 15 -year-olds" has not been achieved yet. In our study, slight differences were detected in the DMFT scores among 12-, 13- and 14-year-olds. In addition, new incidences of caries in primary teeth at the 6- and 12-month examinations were quite low, and no new caries developed at the 18-month examination; moreover, development of any new caries in permanent teeth was not observed at 6,12 , and 18 months.
The epidemiological studies to assess the oral health status in Turkey were carried out in different regions and cities, and thus, different dmft and DMFT scores were obtained.

With regard to the dmft and DMFT index, Öztunç et al. (8) reported $69.2 \%$ caries prevalence in children of the age group 6-11 years residing in Adana, while Altun et al. (9) reported 71.2\% caries prevalence in Ankara. Tulunoğlu et al. (10) reported $2.2 \mathrm{dmft}$ and 0.4 DMFT in children aged 3-8 years in a study conducted in Ankara. Kırzıoğlu et al. (11) reported 2.49, 2.77 and $2.63 \mathrm{dmft}$ index scores in children aged $2-5$ years, respectively. Gökalp et al. (5) reported $3.7 \mathrm{dmft}$ in children aged 5 years, 1.9 DMFT in children aged 12 years, and 2.3 DMFT in children aged 15 years in Erzurum, Bursa, and Isparta, Turkey, in 2004.

A 2010 study including 145 children aged between 3-6 years in the kindergartens of Dokuz Eylül University reported that the percentage of children with dental caries was $29.7 \%$, children with filled tooth $5.5 \%$, and total $\mathrm{dmft}$ score was 0.96 (12).

A study assessing the oral-dental health level of 245 children aged 5-9 years reported the following values: $\mathrm{dmft} 5.3 \pm 3.78$; DMFT $0.27 \pm 0.74$; mean of decayed teeth $3.9 \pm 3.46$; mean of filled teeth $1.0 \pm 1.70$; and mean of missing teeth $0.36 \pm 0.88$ (13).

According to the epidemiological research carried out by Alvarez-Arenal et al. (14) in Spain and Austria, the dmft index of 6-year-olds was 2.10 and the DMFT index was 0.25 , the dmft index of 9-year-olds was 2.38 and DMFT index was 1.5, while the DMFT index of 12-year-olds was 3.30. Jürgensen and Petersen (15) determined that the DMFT index of children aged 12 years in Laos was 1.8 and caries prevalence was $56 \%$. Llompart et al. (16) reported $4.64 \mathrm{dmft}$ and $0.48 \mathrm{DMFT}$, and $70 \%$ caries prevalence in 6-year-olds in Argentina. Although the dmft index scores were lower depending on the oral health education provided by schools, healthcare organizations and media in European countries, as compared to the findings in our study, similar results were obtained considering the DMFT index scores in permanent teeth.

According to the findings of our study, the importance given to primary teeth in patients seeking care at our clinic is much lower when compared to that for permanent teeth, and corroboratively, very high dmft scores were obtained.

Forty-eight percent of children who were included in the study had never been examined by a dentist, and 38\% had been given dental treatment. In a nation-wide study in Turkey, Gökalp et al. concluded that the percentage of 5-year-olds who never went to a dentist was $82.1 \%(5)$.

The results of our study indicated that children do not follow recommendations regarding the frequency of tooth brushing, reflected by a very low percentage $(19 \%)$ of those who brush their teeth no less than twice a day and $56 \%$ of those who reported tooth brushing once a day. The percentage of children who reported no tooth brushing during the day was $18 \%$. These findings show that the children seeking care at our clinic do not act upon the instructions for routine tooth brushing. Moreover, with regard to the habit of brushing teeth in preschool children, our study determined a lower percentage of preschool children compared to school children. Sun et al. (17) showed that only $14.2 \%$ of the total study population were brushing twice per day or more in the primary dentition. The importance of parental brushing and supervision of children until the age of 7 years is also important and should be explored in future research. 
Previous studies have shown that the percentage of caries in children of families with high socioeconomic level was lower than in the children of families with low socioeconomic level $(7,18$, 19). In our study, the data were obtained from the assessment of patients from different socioeconomic levels (low, medium, high) and higher dmft/DMFT scores resulted in the initial examinations of patients belonging to the low socioeconomic subgroup.

Tooth brushing is known to reduce the risk of dental caries. Individuals who brush their teeth at least twice a day and those who adopted this habit at an early age are reported to develop less caries. Moreover, it is suggested that the socioeconomic level of families also affect the adoption of these habits (20).

In the past 20 years, the severity and frequency of dental caries has markedly decreased among children of many developed countries. This decline is mostly associated with the use of fluoride, improved standards of living, dentistry services available for larger populations, as well as mostly increased public awareness on dental health, and informed dental practices. Parents are required to learn some basic issues, such as nutrition, prophylactic measures, and essentials for oral health, for application of the right approach towards dental health of children.

The studies showed that one-time examinations are not adequate in children, however, recurrent dental examinations on a long-term basis and educating children of pre-school age and above on oral and dental health may lead to better results in terms of decreasing the incidences of dental caries $(12,21)$.

\section{CONCLUSION}

In order to raise generations with good oral and dental health in Turkey, it is essential to promote preventive dentistry practices and raise awareness in families. In addition, it is concluded that one-time examinations are not sufficient, and children should be monitored for at least 2 subsequent years and examined twice a year. This study showed that the incidence of dental caries declines in children with periodical examination and preventive dental treatment.

Consequently, paediatric dentists play a significant role in promoting periodical examinations and good dietary habits in patients, providing information on oral hygiene, and providing information to parents and children on the dental treatments.

Providing individuals with information on oral and dental health from elementary grade onward, and regular examinations at a 6-month interval will reduce the caries prevalence combined with early diagnosis and prevention of malocclusions, and contribute to the national economy by avoiding the need for more expensive treatments. Parents, teachers, and dentists play a crucial role in informing children about oral and dental health at an early age in order to raise a healthy population, thereby highlighting the significance of preventive dentistry services prior to the development of dental caries.

\section{Acknowledgement}

This project was supported by Adnan Menderes University, Scientific Research Projects Coordination Unit Number: 2014/DHF-14002.

\section{REFERENCES}

1. Varenne B, Petersen PE, Ouattara S. Oral health status of children and adults in urban and rural areas of Burkina Faso, Africa. Int Dent J. 2004 Apr;54(2):83-9

2. Grewal H, Verma M, Kumar A. Prevalence of dental caries and treatment needs in the rural child population of Nainital District, Uttaranchal. J Indian Soc Pedod Prev Dent. 2009 Oct-Dec;27(4):224-6.

3. Gökalp SG, Doğan BG, Tekçiçek MT, Berberoğlu A, Unlüer S. National survey of oral health status of children and adults in Turkey. Community Dent Health. 2010 Mar;27(1):12-7.

4. Shah AF, Tangade P, Ravishankar TL, Tirth A, Pal S, Batra M. Dental caries status of institutionalized orphan children from Jammu and Kashmir, India. Int J Clin Pediatr Dent. 2016 Oct-Dec;9(4):364-71.

5. Gökalp S, Güçiz Doğan B, Tekçiçek M, Berberoğlu A, Ünlüer Ș. The oral health profile of 5, 12 and 15 year olds, Turkey-2004. J Hacettepe Fac Dent. 2007;31(4):3-10. (In Turkish.)

6. Güçiz Doğan B, Gökalp S. Caries status and treatment needs in Turkey, 2004. J Hacettepe Fac Dent. 2008;32(2):45-57. (In Turkish.)

7. Saydam G, Oktay İ, Möller I. Oral health in Turkey - situation analysis İstanbul: WHO Regional Office for Europe; 1990. (In Turkish.)

8. Öztunç H, Haytaç MC, Özmeriç N, Uzel İ. The evaluation of oral health status of 6-11- year-old children in Adana (Adana DSİ Primary School, 1999). Gazi Univ Hekim Fak Derg. 2000;17(2):1-6. (In Turkish.)

9. Altun C, Güven G, Başak F, Akbulut E. Evaluation of children in the age group of 6 to 11 with respect to oral-dental health. Gülhane Med J. 2005;47(2):114-8. (In Turkish.)

10. Tulunoğlu Ö, Bodur H, Ulusu T, Ciğer R, Odabaş M. A comparative evaluation of caries prevalence and distrubition of tooth surface of preschool (3-6 age) and school children (7-8 age). Gazi Univ Hekim Fak Derg. 2003;20(3):11-6. (In Turkish.)

11. Kırzıoğlu Z, Gürbüz T, Şimşek S, Yağdıran A, Karatoprak O. Prevalence of caries in children aged 2-5 years and evaluation of some risk factors in Erzurum, Bursa and Isparta Provinces. Atatürk Univ Hekim Fak Derg. 2002;12(2):6-13. (In Turkish.)

12. Kılınç G, Koca H, Ellidokuz H. The oral treatments clinical follow-up For 2 years on the 3-4 years old children at Dokuz Eylul University's Kindergarden. Dokuz Eylül Üniversitesi Tıp Fakültesi Dergisi. 2013;27(1):2531. (In Turkish.)

13. Köksal E, Tekçiçek M, Yalçın SS, Tuğrul B, Yalçın S, Pekcan G. Association between anthropometric measurements and dental caries in Turkish school children. Cent Eur J Public Health. 2011;19(3):147-51.

14. Alvarez-Arenal A, Alvarez-Riesgo JA, Peña-Lopez JM, FernandezVazquez JP. DMFT, dmft and treatment requirements of schoolchildren in Asturias, Spain. Community Dent Oral Epidemiol. 1998;26(3):166-9.

15. Jürgensen N, Petersen PE. Oral health and the impact of socio-behavioural factors in a cross sectional survey of 12-year old school children in Laos. BMC Oral Health. 2009 Nov 16;9:29. doi: 10.1186/1472-6831-9-29.

16. Llompart G, Marin GH, Silberman M, Merlo I, Zurriaga O, GIS (Grupo Interdisciplinario para Salud). Oral health in 6-year-old school children from Berisso, Argentina: falling far short of WHO goals. Med Oral Patol Oral Cir Bucal. 2010;15(1):e101-5.

17. Sun X, Bernabé E, Liu X, Gallagher JE, Zheng S. Early life factors and dental caries in 5-year-old children in China. J Dent. 2017 Sep;64:73-9.

18. Jenny J, Cons NC. Comparing and contrasting two orthodontic indices, the Index of Orthodontic Treatment Need and the Dental Aesthetic Index. Am J Orthod Dentofacial Orthop. 1996 Oct;110(4):410-6.

19. Ainamo J, Barnes D, Beagrie G, Cutress T, Martin J, Sardo-Infirri J. Development of the World Health Organization (WHO) community periodontal index of treatment needs (CPITN). Int Dent J. 1982 Sep;32(3):281-91.

20. Waldman HB. Do parents know how best to feed their children? Maybe not! ASDC J Dent Child. 1998 Sep-Oct;65(5):335-8, 355.

21. Yıldız E, Şimşek M, Gündoğar Z, Aktan AM. Oral health survey of children referring to Faculty of Dentistry in Gaziantep. Gaziantep Med J. 2015;21(2):118-24. (In Turkish.)

\section{Conflict of Interests}

None declared 